Research Paper

\title{
A 40-50klDa Glycoprotein Associated with Mucus is Identified as $\alpha$-l-Acid Glycoprotein in Carcinoma of the Stomach
}

\author{
Nthato Chirwa ${ }^{1}$, Dhirendra Govender ${ }^{2}$, Bongani Ndimba ${ }^{3}$, Zoe Lotz ${ }^{1}$, Marilyn Tyler ${ }^{1}$, Eugenio Panieri', \\ Delawir Kahn ${ }^{1}$ and Anwar S Mall ${ }^{1 \times}$
}

1. Department of Surgery, University of Cape Town, South Africa;

2. Department of Anatomical Pathology, University of Cape Town, South Africa;

3. Proteomics Research Group, Department of Biotechnology, University of the Western Cape, South Africa.

$\triangle$ Corresponding author: Anwar Suleman Mall PhD, Department of Surgery, University of Cape Town, J-Floor, Old Main Building, Groote Schuur Hospital, Observatory 7925, South Africa. Tel: +27 21 406-6232; Fax: +27 21 448-6461; Email: anwar.mall@uct.ac.za

( ) Ivyspring International Publisher. This is an open-access article distributed under the terms of the Creative Commons License (http://creativecommons.org/ licenses/by-nc-nd/3.0/). Reproduction is permitted for personal, noncommercial use, provided that the article is in whole, unmodified, and properly cited.

Received: 2011.10.13; Accepted: 2012.12.05; Published: 2012.02.09

\begin{abstract}
Background and Aim: Secreted gastric mucins are large O-glycosylated proteins of crude mucus gels which are aberrantly expressed in malignancy. An albumin associated $55-65 \mathrm{kDa}$ glycoprotein was previously shown in mucus gels in gastric cancer. The aim of this study was to investigate its expression and identification in human gastric tissue. Methods: Mucins were purified from crude mucus scrapings of 16 partial and II total resections and a rabbit polyclonal antibody was raised to the $55-65 \mathrm{kDa}$ glycoprotein. The location and expression of the glycoprotein was examined in normal gastric mucosa $(n=20)$, intestinal metaplasia $(n=18)$ and gastric cancer $(n=27)$ tissue by immunohistochemistry. Mucins were analyzed by isoelectric focusing (IEF) on 2-D polyacrylamide gels. Identification of the $40-50 \mathrm{kDa}$ glycoprotein was by MALDI-TOF MS technique. Plasma levels were examined by Western blotting. Results: Extensive SDS-PAGE analysis gave a PAS positive glycoprotein in the $40-50 \mathrm{kDa}$ range, in patients with gastric cancer but not normals. It was expressed in parietal and columnar cells of normal gastric tissue and intestinal metaplasia respectively, and in 22 of 27 gastric cancer specimens. In 2-D PAGE stained with Coomassie Blue there were 3 spots positively identified as alpha-I-acid glycoprotein (AGP) by MALDI-TOF MS technique. PAS staining revealed a single bright spot in the same position but could not be identified. Preliminary measurements showed slightly higher levels of AGP in plasma of patients with gastric carcinoma. Conclusion: AGP levels are increased in gastric tissue and in the plasma of those with carcinoma of the stomach.
\end{abstract}

Key words: Mucus, Mucin, intestinal metaplasia, gastric, cancer, a-1-acid-glycoprotein.

\section{Introduction}

The gastrointestinal tract is coated with a protective visco-elastic mucus gel synthesized and secreted by specialized cells in the surface and/or underlying submucosa. ${ }^{1}$ In the normal gastric mucosa, MUC12, MUC4 ${ }^{3}$, MUC5AC $^{4}$ and MUC6 5 are expressed in a characteristic zonal pattern. MUC1 has a membranous staining pattern in the surface epithelium and mucus neck zone cell ${ }^{2}$ whilst MUC4 stained in both normal stomach tissue and gastritis ${ }^{3}$. The distribution of MUC5AC is limited to the cytoplasm of the surface epithelium and mucus neck cells. ${ }^{6}$ MUC6 is expressed in the cytoplasm of the deep mucus glands and mucus neck cells. ${ }^{5}$ The gastric mucus gel, composed of MUC5AC and MUC6, ${ }^{5}$ protects the gastric epithelia 
from the shear forces associated with digestion. ${ }^{7}$

Gastric cancer, a fatal malignancy with 700,000 deaths reported annually ${ }^{8}$ is prevalent in the Western Cape region of South Africa among so-called Cape Coloured males (a hybrid race of Western European, Southern African and Asian origin). ${ }^{9-11}$ Clinical markers for early detection of disease include carcinoembryonic antigen (CEA) and CA 19-9, both of which lack specificity ${ }^{12,13}$ and in the case of CA 19-9, detectable more often in the advanced stage of gastric carcinoma than in the early stages. ${ }^{14}$ There has been an increasing interest in mucins in the detection and treatment of carcinomas in general ${ }^{15}$ in particular as diagnostic and therapeutic agents. ${ }^{16}$

Both our laboratory ${ }^{17-21}$ and another ${ }^{22}$ reported the presence of a clinically useful marker in the crude mucus scrapings and gastric juice of gastric cancer patients. It was suggested that this albumin associated glycoprotein, previously estimated to be of $\mathrm{M}_{\mathrm{r}} \sim$ $55-65 \mathrm{kDa}$ and now $\mathrm{M}_{\mathrm{r}} \sim 40-50 \mathrm{kDa}$ with extensive SDS-PAGE analysis, was a fragment of MUC5AC. ${ }^{20}$ In this study the location and expression of this glycoprotein and mucins in gastric mucosa were studied and its identification as a-1-acid glycoprotein (AGP), an acute phase protein, established. The levels of AGP were preliminarily shown to be slightly higher in the bloods of patients undergoing resection for carcinoma of the stomach, than in normals.

\section{Materials and Methods}

\section{Ethics and Patients}

The University of Cape Town Research Ethics Committee approved this study (REC REF: 084/2002). Twenty seven gastrectomy specimens (16 partial and 11 total resections) were obtained from patients who had undergone surgery between 2005 and 2008 at Groote Schuur Hospital $(n=23)$ and the University of Cape Town Private Academic Hospital $(n=4)$.

\section{Collection preparation and analysis of gastric mucus}

Crude mucus was scraped from the gastrectomy specimens, solubilized by brief homogenization in ice-cold $6 \mathrm{M}$ guanidinium $\mathrm{HCl}$ containing a cocktail of protease inhibitors, and purified by density gradient centrifugation in $\mathrm{CsCl}$ twice, as described previously. ${ }^{20,23}$ Approximately 500 $\mu \mathrm{g}$ purified mucin was loaded on $10 \%$ SDS-PAGE followed by Western blotting performed as described by Mall et al. ${ }^{24}$ Nitrocellulose membranes were probed with the polyclonal antibody to the $40-50 \mathrm{kDa}$ glycoprotein raised in a three month old female rabbit by the method of Bellstedt et al. 25

\section{Histochemistry and Immunohistochemistry}

Formalin fixed paraffin wax embedded gastric tissue blocks (matched to the patients that had mucus scraped from their stomachs) were obtained from the archives of the Division of Anatomical Pathology, National Health Laboratory Service (NHLS) - Groote Schuur Hospital. All sections $(2 \mu \mathrm{m})$ were routinely stained for haematoxylin and eosin and special stains for mucin, PAS/AB and HID/AB and assessed by a pathologist (DG). The samples included 3 normal mucosa sampled away from the tumour, 17 normal mucosa immediately adjacent to tumour, 13 intestinal type carcinomas, 7 diffuse carcinomas, 1 mixed intestinal type and diffuse carcinoma, 4 signet ring carcinomas and 2 mucinous carcinomas.

Immunohistochemistry was done with primary rabbit polyclonal antibody against the 40-50kDa glycoprotein (diluted in PBS and HSA), monoclonal mouse anti - MUC1, MUC1core, MUC2, MUC4, MUC5AC and MUC6 and rabbit anti-human-albumin polyclonal antibody, diluted in PBS, according to Mall et al. ${ }^{26}$ Normal small intestine was used as a positive control for the $40-50 \mathrm{kDa}$ glycoprotein, normal stomach for MUC1, normal colon for MUC2 and MUC4 and normal stomach for MUC5AC and MUC6. PBS was used instead of the primary antibodies for negative control. Sections were then incubated with the respective secondary antibody (Envision anti-rabbit HRP or anti-mouse HRP) and detection of staining using DAB.

\section{D SDS PAGE of mucin-rich sample prepara- tions}

This was a slight modification to that described by Thomas et al. ${ }^{27}$ Protein $(100 \mu \mathrm{g}$ for CBB or $200 \mu \mathrm{g}$ for PAS staining), in the mucin-rich preparations, was precipitated using TCA/acetone, air dried and then resolubilized to a final volume of $125 \mu \mathrm{L}$ in urea buffer (9M urea, 2M thiourea, $4 \%(\mathrm{w} / \mathrm{v})$ 3-[(3cholamidopropyl) dimethylammonio]-1-propanesulfonate (CHAPS) and mixed with $0.8 \%(\mathrm{w} / \mathrm{v})$ dithiothreitol (DTT), $0.2 \% \quad(\mathrm{v} / \mathrm{v})$ ampholytes $\mathrm{pH} \quad 3-10$ (BIO-RAD, Hercules, CA, USA), with $0.002 \%$ bromophenol blue. The samples were then used to passively rehydrate $7 \mathrm{~cm}$ IPG linear strips, $\mathrm{pH}$ 3-10 (BIO-RAD), overnight at room temperature. The strips were subjected to isoelectric focusing (IEF) using the EttanTM IPGphor IITM (GE Healthcare, Amersham, UK), in a step-wise programme for a total of $12,000 \mathrm{Vh}$ at $20^{\circ} \mathrm{C}$ for $6 \mathrm{~h}$. Prior to the second dimension, the strips were equilibrated twice for $10 \mathrm{~min}$ with gentle shaking in an equilibration buffer $(6 \mathrm{M}$ urea, 2\% (w/v) SDS, 0.05M Tris- $\mathrm{HCl}, \mathrm{pH} 8.8,20 \%$ $(\mathrm{v} / \mathrm{v})$ glycerol and $0.002 \%$ bromophenol blue) con- 
taining $1 \%(\mathrm{w} / \mathrm{v})$ DTT and then $2.5 \%(\mathrm{w} / \mathrm{v})$ iodoacetamide. The strips were then loaded on a $12 \%$ SDS-PAGE and electrophoresed vertically at $120 \mathrm{~V}$ for 90 min. The gels were stained with CBB or PAS and imaged with the PharosFXTM plus molecular imager scanner (BIO-RAD).

\section{In-gel digestion and MALDI-TOF MS identifi- cation of protein spots from 2-D gels}

The CBB and PAS stained 2-D PAGE spots were excised and manually plugged out with sterile cut pipette tips and placed into sterile micro-centrifuge tubes. Proteins in gel plugs (approximately $2 \mathrm{~mm}$ in diameter and $1 \mathrm{~mm}$ in height) were trypsinised and processed for identification according to the method of Thomas et al. ${ }^{27}$

Western blots were done of blood samples obtained from healthy donors $(n=49)$ and patients undergoing gastrectomy for cancer $(n=24)$ and probed with antibody against a-1-acid glycoprotein (AGP).

\section{Results}

\section{Preparation and analysis of purified mucin}

There was a clear separation of mucin from contaminating proteins after density gradient centrifugation in $\mathrm{CsCl}$ (result not shown). The purified mucin was run on two gels, one stained with PAS
(Figure 1A) to confirm the presence of the $40-50 \mathrm{kDa}$ glycoprotein (Figure 1A, lane 6, circled) and mucins (Figure 1A, lane 7, arrow), and the other with Ponceau $S$ (Figure 1B) from which the 40-50kDa glycoprotein was cut just below the region where albumin (Figure $1 \mathrm{~B}$, lane 1 , arrow) was visible. In both gels, BSA was loaded in lanes 1 and stained positively with Ponceau $\mathrm{S}$ (Figure 1B) and not with PAS (Figure 1A).

A strong reactivity to the $40-50 \mathrm{kDa}$ glycoprotein was seen in purified mucin from a gastric cancer specimen (Figure 2A, lane 6 arrow). Cross-reactivity with albumin (Figure 2A, lanes: 1-5 and 7-8) was abolished with incubation of the antibody with HSA prior to electrophoresis leaving reactivity only to cancer mucin, presenting as a smear at the top of the running gel (Figure 2B, lanes 6 and 9, arrowhead), suggesting non-specific binding of this glycoprotein with mucins or the presence of similar epitopes in both species.

In the lanes which were positive for the 40-50kDa glycoprotein (Figure 2B, lanes 6 and 9), no reactivity was noted in the region of albumin at $66-70 \mathrm{kDa}$ (lane 9, middle arrowhead) with clear reactivity to the 40-50 kDa glycoprotein (lane 9, bottom arrowhead). Very faint material was seen below the $\mathrm{M}_{\mathrm{r}} \sim 40-50 \mathrm{kDa}$ band.

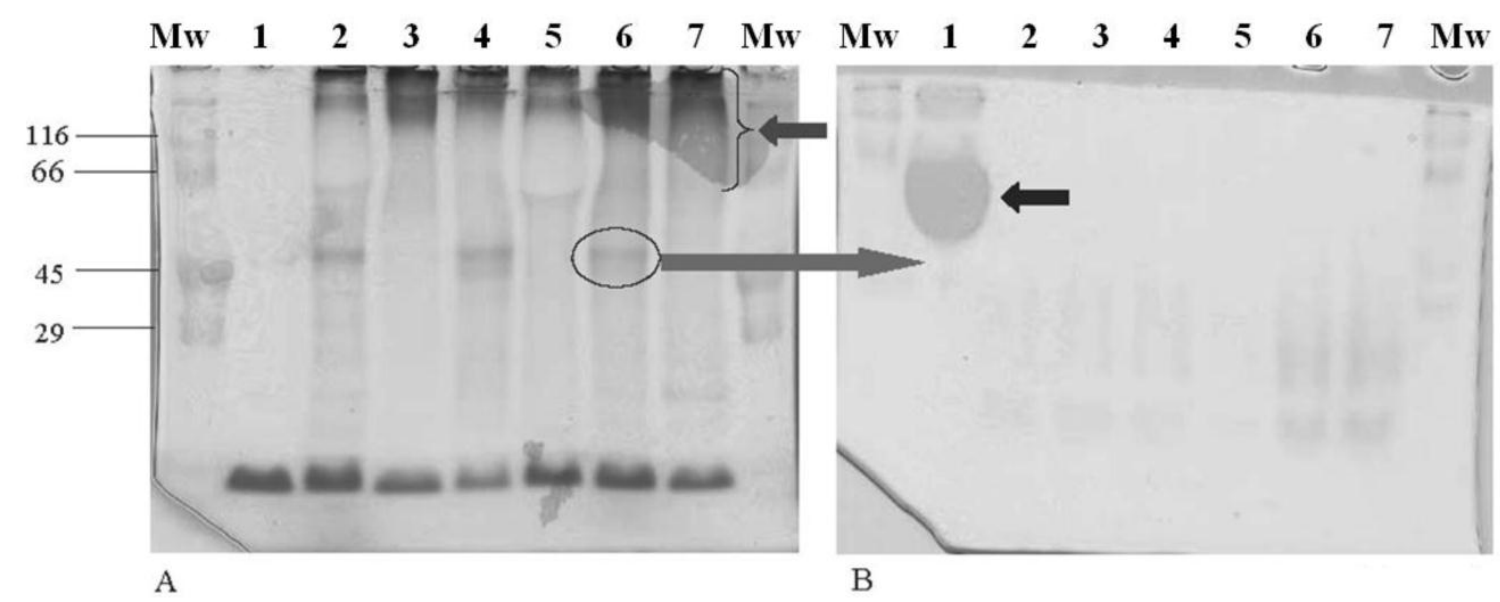

Figure I. Analysis by SDS-PAGE of purified mucin obtained from specimens resected for gastric cancer. Freeze-dried mucin $(500 \mu \mathrm{g})$ from patients with gastric cancers was prepared in reducing sample application buffer and separated on I0\% SDS-PAGE. (A) Gel stained with PAS showing the 40-50 kDa glycoprotein (lane 6, circled) and mucin (lane 7, arrow). (B) Gel stained with Ponceau S showing the "blob" of albumin (lane I, arrow). Gels (A) and (B) contain samples in the following order: Lane I (200 $\mu \mathrm{g}$ BSA), lane 2 (M, $55 y r$, partial gastrectomy, iac), lane 3 (M, 53yr, partial gastrectomy, iac), lane 4 (F, 74yr, partial gastrectomy, dac), lane 5 (M, $60 y r$, partial gastrectomy, srac), lane 6 (F, 57yr, total gastrectomy, dac), lane $7\left(\mathrm{M}, 60 \mathrm{yr}\right.$, partial gastrectomy, dac). The cut gel was stored at $-20^{\circ} \mathrm{C}$ until immunization. (Abbreviations: $M$ - male, $F$ - female, iac - intestinal type carcinoma, dac - diffuse carcinoma, srac - signet-ring cell carcinoma) 

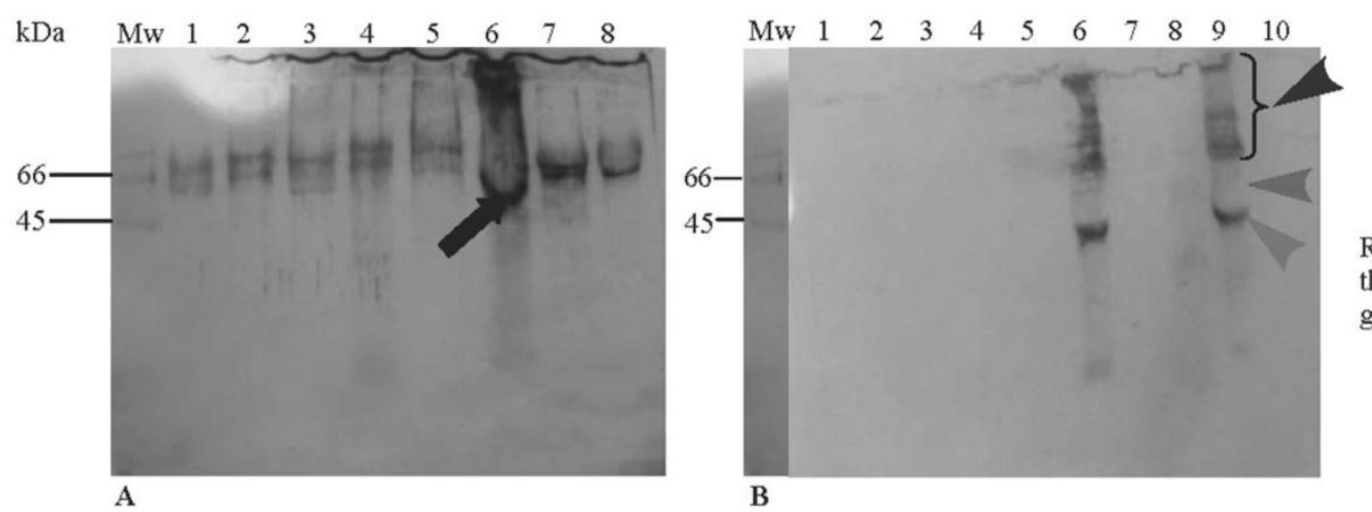

Reactivity

with cancer

mucin

No reactivity with albumin

Reactivity with the $40-50 \mathrm{kDa}$ glycoprotein

Figure 2. Western blot of the 40-50 kDa glycoprotein. Following two $\mathrm{CsCl}$ density gradient steps, purified mucins (300-500 ug) and BSA (190-200 ug) were electrophoresed on SDS-PAGE gel (3\% stacking gel and 10\% running gel). After electrophoresis, proteins were transferred onto a nitrocellulose membrane using a semi-dry electroblotting unit for Ih. Membranes were probed using the antibody to the 40-50 kDa glycoprotein. Immunoreactivity was detected using an ECL kit and films were processed using a standard X-ray developer. (A) Primary anti-(40-50 kDa glycoprotein) antibody prepared without human serum albumin (HSA) and showing high cross reactivity. Lane I (crude PMP), lane 2 (crude PCC), lane 3 (crude PMP), lane 4 (purified FAP), lane 5 (CGC), lane 6 (PGC), lanes 7 and 8 (BSA). (B) Primary anti-(40-50 kDa glycoprotein) antibody incubated with HSA showing no cross-reactivity. Lane I (BSA), lane 2 (PCC), lane 3 (CGC), lane 4 (PGC), lane 5 (crude gastric cancer), lane 6 (PGC), lane 7 (purified PMP), lane 8 (purified FAP), lane 9 (PGC) and lane 10 (BSA). (Abbreviations: PMP - pseudomyxoma peritonei, PCC - purified colon cancer, FAP - familial adenomatous polyposis coli, CGC - crude gastric cancer, PGC - purified gastric cancer and BSA - bovine serum albumin)

\section{Immunohistochemical expression of the 40-50kDa glycoprotein and mucin in normal gastric tissue, intestinal metaplasia and gastric cancer}

The 40-50kDa glycoprotein was consistently expressed in the cytoplasm of parietal cells $(20 / 20)$ (Figure 3A, arrows). None of the mucin antibodies used in this study stained parietal cells. MUC1 was consistently expressed in the surface epithelium, mucus neck cells and in the deep gastric mucus glands (26/26). There was inconsistent staining of MUC4 in the surface epithelium (10/26) and in the deep mucus glands (5/26). MUC5AC consistently stained the surface epithelium (26/26). MUC6 was consistently expressed in the deep mucus glands $(25 / 25)$. MUC1 core and MUC2 were not expressed in the normal gastric mucosa.

Complete intestinal metaplasia was present in 11 of the 12 cases and incomplete intestinal metaplasia was seen in 6 of the 12 cases. Five cases showed both complete and incomplete metaplasia and one case had only incomplete metaplasia. In intestinal metaplasia, the $40-50 \mathrm{kDa}$ glycoprotein was consistently expressed in the columnar cells in complete (type I) IM (11/11), incomplete (type II and III) IM (6/6), (Figure 3B). The results for mucin in IM are as follows: in the complete (type I) IM there was MUC2 (11/11) (goblet cells only) and no MUC1, MUC4, MUC5AC and MUC6. In the incomplete IM there was MUC2 (6/6) (goblet cells), MUC2 (3/6) (columnar cells), MUC5AC (6/6) (co- lumnar cells) and MUC6 (2/6) (columnar cells) with no MUC1 and MUC4.

The 40-50kDa glycoprotein was consistently expressed in the intestinal type carcinoma (13/13) (Figure 3C) and was inconsistently expressed in the diffuse carcinoma (4/7), both showing cytoplasmic staining (Figure 3D). In the mixed (intestinal and diffuse) carcinoma (1/1), the 40-50kDa glycoprotein was expressed in both components. It was also expressed in the signet-ring cell (2/4) and mucinous (2/2) carcinomas.

MUC1 was expressed in the intestinal (12/13), diffuse $(7 / 7)$ type carcinomas, in both components of the mixed (1/1) (intestinal and diffuse), signet-ring cell $(4 / 4)$ and in the mucinous carcinomas (2/2). MUC1 core was expressed in the intestinal (10/13), diffuse $(1 / 7)$, in both components of the mixed (1/1), in the signet-ring cell $(2 / 4)$ and in the mucinous carcinomas $(2 / 2)$. MUC2 was expressed in the intestinal $(2 / 13)$, diffuse $(2 / 7)$, signet-ring cell $(3 / 4)$ and in the mucinous carcinomas $(1 / 2)$ and not in the mixed carcinoma. MUC4 was expressed in the intestinal type $(8 / 13)$, in the diffuse type $(2 / 7)$ and in both components of the mixed (1/1) carcinomas and not in the signet-ring cell and mucinous carcinomas. MUC5AC was expressed in the intestinal type (6/13), in the diffuse type (3/7) and in the signet-ring cell carcinomas $(3 / 4)$ and not in the mixed and in the mucinous carcinomas. MUC6 was expressed in the intestinal type $(4 / 13)$, in the diffuse type $(1 / 7)$ and in the signet-ring cell carcinomas $(1 / 4)$ but not in the mixed 
and the mucinous carcinomas.

Normal stomach expressed neutral mucins. There was a decrease of neutral mucin in IM with sialomucins predominant in the complete IM and both sialomucins and sulphomucins in the incomplete IM (results not shown).

Three (spots 1-3) well-resolved and closely aligned spots with approximate molecular weights of $50 \mathrm{kDa}$ and a $\mathrm{pI}$ between 3 and 4 were detected after CBB staining of the 2D gel (Figure $4 \mathrm{~A}$ ).

The two spots resolved on the more acidic side of the gel were of greater abundance than the third spot. The three spots differ slightly, not only in their pI, but also in their molecular weight, with the most acidic spot displaying a slightly higher mass than the second spot and the second one higher than the third. Following PAS staining, only one single spot (spot 4) with a molecular weight of approximately $50 \mathrm{kDa}$ and a pI close to 4 was visualized on the 2D gel (Figure 4B).

\section{Detection of total proteins and glycoproteins from mucin samples by 2-D gel electrophoresis}

\section{Protein identification by MALDI-TOF MS}

The list of experimental peptide masses was compared against the theoretical tryptic digest of every protein in the MSDB and NCBI databases through the MASCOT software. Spots 1, 2 and 3 were positively identified, as defined by significant probability-based MOWSE scores between 67-73 as human alpha-1-acid glycoprotein (AGP; Table 1). Although spot 4 digested well, as shown and defined by the abundance of peptides and a high signal to noise ratio, the MOWSE score obtained was lower than 64, which falls below the threshold, and the protein was thus not positively identified. When comparing the list of protein peptides obtained from spots 1, 2 and 3 (Figure 4) with the peptides from spot 4 (Table 2), the profiles looked similar with at least 10 identical peptide masses, highlighted with a bold font. Tryptic digest peptide masses obtained three spots 1, 2 and 3 which were all positively matched to alpha-1-acid glycoprotein 1 precursor. The inability to obtain a positive hit from tryptic digest peptides generated from spot 4 suggests that the PAS protein staining is incompatible with the MALDI-TOF mass spectrometric method of protein identification.

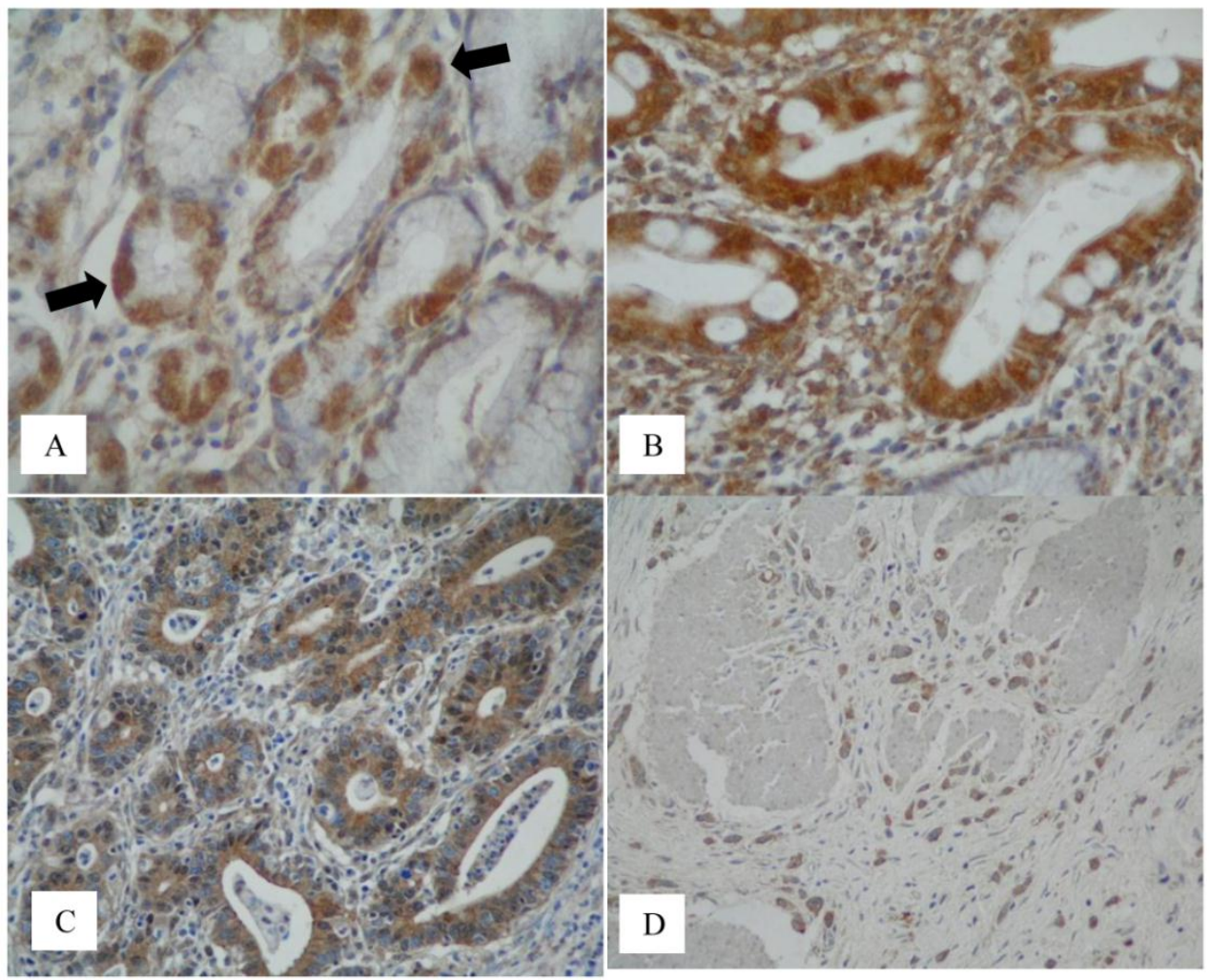

Figure 3. Immunodetection of the 40-50 kDa glycoprotein in normal, intestinal metaplasia and gastric cancer tissue. (A) Extensive staining of parietal cells ( $x 40$, black arrows) was consistently seen on normal tissue sections (normal adjacent to tumour) stained with the anti-(40-50 kDa glycoprotein) antibody. This was also observed in the control tissues sampled away from cancer. (B) The 40-50 $\mathrm{kDa}$ glycoprotein was expressed in the cytoplasm of columnar cells in complete and incomplete (not shown) IM but not in the goblet cells (x40). (C) The 40-50 kDa glycoprotein was expressed in the cytoplasm of neoplastic glands in the intestinal type carcinoma (x40) and (D) by cancer cells of the diffuse type shown invading the muscle wall of the stomach $(x 20)$. 


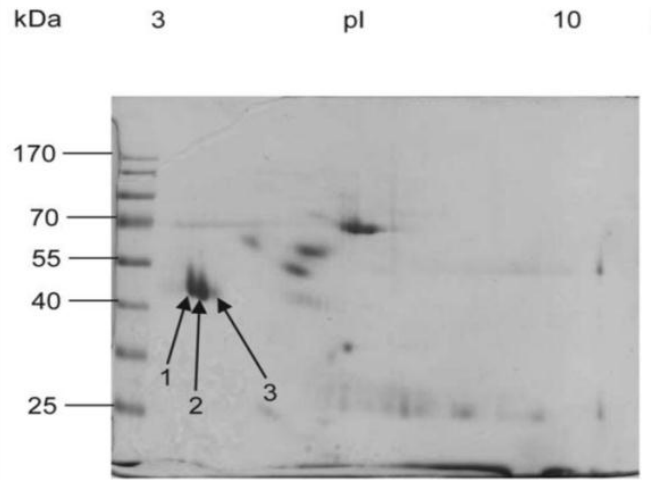

A
kDa 3

pl

10

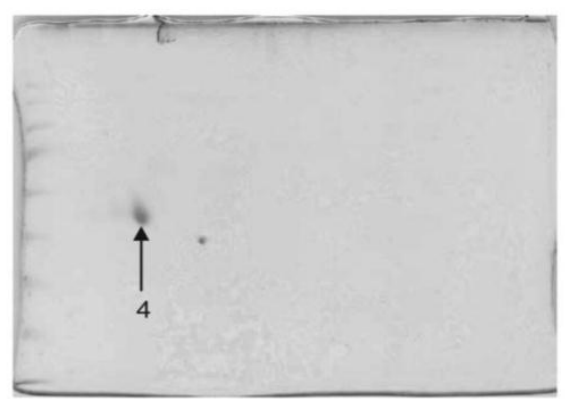

B

Figure 4. Two-dimensional gel electrophoresis of purified mucin protein sample from gastric cancer specimens. Purified mucin sample (100 $\mu \mathrm{g}$ for CBB and $200 \mu \mathrm{g}$ for PAS) from gastric cancer specimens was separated using linear $7 \mathrm{~cm} \mathrm{IPG} \mathrm{strips,} \mathrm{pH} \mathrm{range}$ 3- 10 in the first dimension and I2\% SDS-PAGE in the second dimension. Gels were then stained with (A) Coomassie Brilliant Blue showing three protein spots (arrows I, 2 and 3). (B) PAS showing one glycoprotein spot (arrow 4).
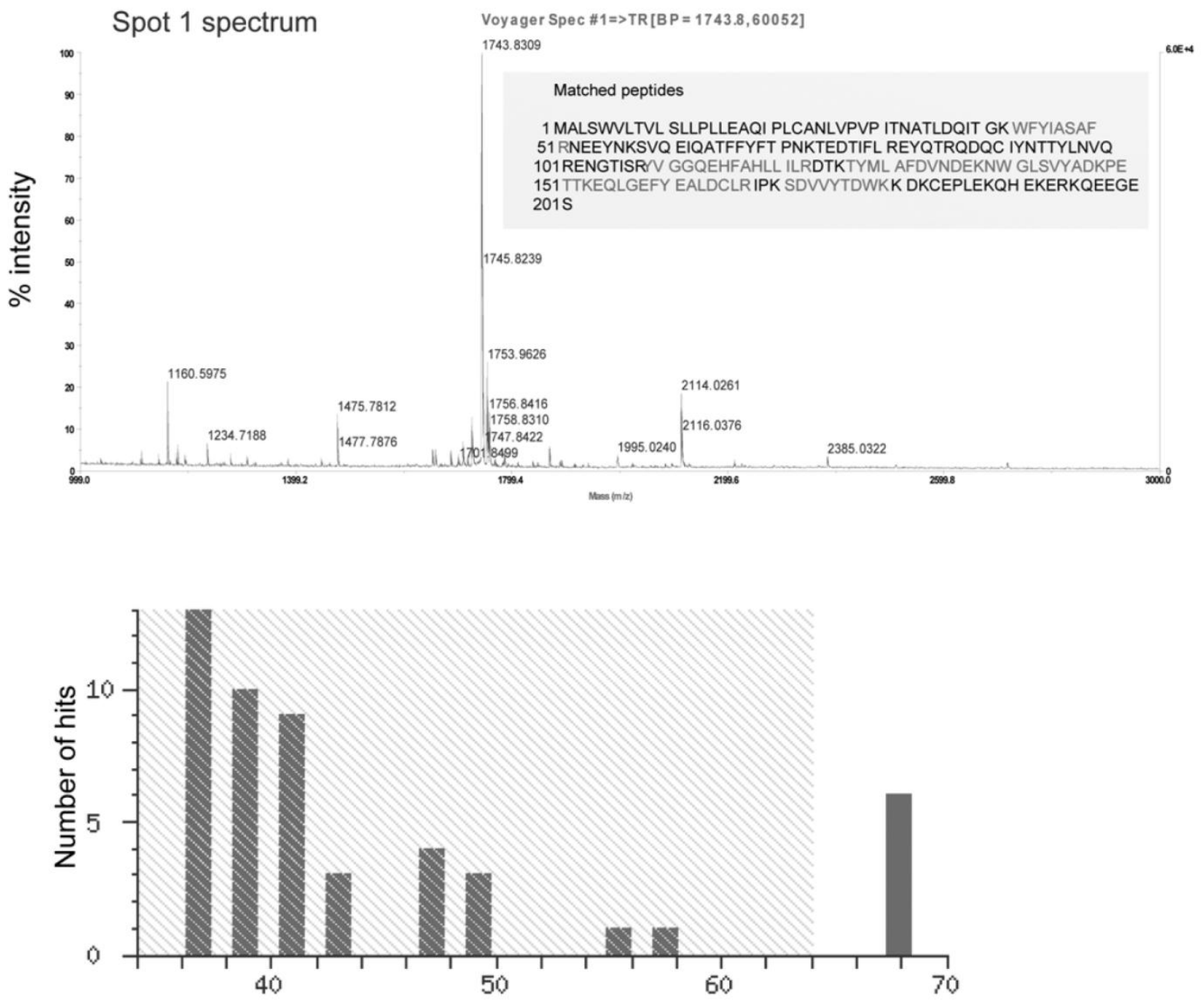

Probability Based Mowse Score for spot 1

Figure 5. Mass spectra obtained by MALDI-TOF MS for the digested protein spots visualized on 2D gel after CBB staining. After CBB staining of the 2D gel of purified mucin protein samples from gastric cancer individuals, spots I, 2 and 3 (Figure 4A) were manually excised from the gel, digested with trypsin, and analyzed by MALDI-TOF MS for spectrum acquisition. Using the mass of the obtained peptides, the proteins were identified using the Mascot database. Spectra obtained for spots I, 2 and 3 were similar. 
Table I. Recapitulative table of protein spots identified by MALDI-TOF MS. Mucin proteins were identified by MALDI-TOF-MS. The table lists the spot number, corresponding to the numbers in Figure 5, accession number, protein name, species, theoretical and observed molecular weights $(\mathrm{kDa})$ and $\mathrm{pl}$, MOWSE score (number greater than 64 being significant at $p<0.05$ ) and the sequence coverage of the positive identification.

\begin{tabular}{lllllll}
\hline $\begin{array}{l}\text { Protein Spot } \\
\text { number }\end{array}$ & $\begin{array}{l}\text { Pl from data- } \\
\text { base }\end{array}$ & Pl from gel & $\begin{array}{l}\text { Molecule weight } \\
\text { from database (kDa) }\end{array}$ & $\begin{array}{l}\text { Molecule weight } \\
\text { from gel (kDa) }\end{array}$ & $\begin{array}{l}\text { Mowse } \\
\text { Score }\end{array}$ & Name of protein identified \\
\hline 1 & 4.92 & $3-4$ & 46.994 & $40-50$ & 68 & $\begin{array}{l}\text { Alpha-1-acid glycoprotein 1 precursor } \\
\text { [validated]-human } \\
\text { Alpha-1-acid glycoprotein 1 precursor } \\
\text { [validated]-human } \\
\text { Alpha-1-acid glycoprotein 1 precursor } \\
\text { [validated]-human }\end{array}$ \\
\hline
\end{tabular}

Table 2. Tryptic peptide mass peak list for each spot analyzed by MALDI-TOF MS. Coomassie (spot I-3) and PAS stained (spot 4) protein were analyzed by MALDI-TOF MS. The table lists tryptic digest peptide masses $(\mathrm{m} / \mathrm{z})$ for each of the four protein spots. Masses shared by spot 4 and at least one other from the spots identified as AGP (spot I-3).

\begin{tabular}{|c|c|c|c|}
\hline Spot 1 & Spot 2 & Spot 3 & Spot 4 \\
\hline & & & 1002.5854 \\
\hline \multicolumn{4}{|l|}{1112.5379} \\
\hline \multirow[t]{2}{*}{1144.5245} & & & 1144.6054 \\
\hline & & & 1158.6726 \\
\hline \multirow[t]{2}{*}{1160.5976} & 1160.6009 & 1160.6106 & 1160.6790 \\
\hline & & & 1164.6668 \\
\hline \multirow[t]{2}{*}{1176.5922} & & & 1176.6702 \\
\hline & & 1179.6230 & 1179.6781 \\
\hline 1192.5896 & 1192.5866 & & 1192.6728 \\
\hline 1234.7168 & & 1234.7171 & 1234.7904 \\
\hline \multicolumn{4}{|l|}{1277.7423} \\
\hline 1307.6946 & & 1307.7095 & \\
\hline \multicolumn{4}{|l|}{1445.6883} \\
\hline & & & 1461.8276 \\
\hline \multirow[t]{2}{*}{1475.7815} & 1475.7568 & 1475.7768 & \\
\hline & & & 1633.8864 \\
\hline 1651.8197 & 1651.7785 & & 1651.9035 \\
\hline \multirow[t]{2}{*}{1657.8168} & & 1657.8257 & 1657.9082 \\
\hline & & & 1668.9096 \\
\hline 1685.8064 & 1685.7597 & 1685.7997 & 1685.9017 \\
\hline \multicolumn{4}{|l|}{1699.8418} \\
\hline & & & 1701.8680 \\
\hline & & & 1703.8405 \\
\hline & & 1707.7905 & \\
\hline 1708.8478 & 1708.7901 & 1708.7954 & \\
\hline \multicolumn{4}{|l|}{1716.8704} \\
\hline 1724.8238 & 1724.7471 & 1724.7937 & \\
\hline \multicolumn{4}{|l|}{1725.8136} \\
\hline 1726.7949 & & 1726.7877 & \\
\hline 1742.8295 & 1742.7520 & 1742.8043 & 1741.8838 \\
\hline 1744.0956 & & & \\
\hline
\end{tabular}

\begin{tabular}{|c|c|c|c|}
\hline Spot 1 & Spot 2 & Spot 3 & Spot 4 \\
\hline & & 1746.8055 & \\
\hline 1752.9635 & 1752.8899 & 1752.9530 & 1753.0573 \\
\hline \multirow[t]{3}{*}{1756.8420} & 1756.7619 & 1756.8323 & \\
\hline & & & 1758.9559 \\
\hline & & & 1842.0540 \\
\hline \multicolumn{4}{|l|}{1784.9557} \\
\hline 1868.7568 & & 1868.7870 & \\
\hline \multicolumn{4}{|l|}{1888.0296} \\
\hline & & & 1931.0856 \\
\hline & & & 1936.9104 \\
\hline \multirow[t]{9}{*}{1994.0182} & & 1940.9774 & \\
\hline & 2021.8990 & & \\
\hline & 2094.8900 & & \\
\hline & 2096.9331 & & \\
\hline & 2112.9138 & & \\
\hline & 2126.9287 & & \\
\hline & 2021.8990 & & \\
\hline & & 2225.1416 & \\
\hline & & 2239.1893 & \\
\hline \multicolumn{4}{|l|}{2384.0306} \\
\hline & & 2510.1669 & \\
\hline 2717.1665 & & & \\
\hline
\end{tabular}


Preliminary studies show that blood levels of AGP are variably increased in patients pre-operatively compared to levels in normal bloods (Figure 6).

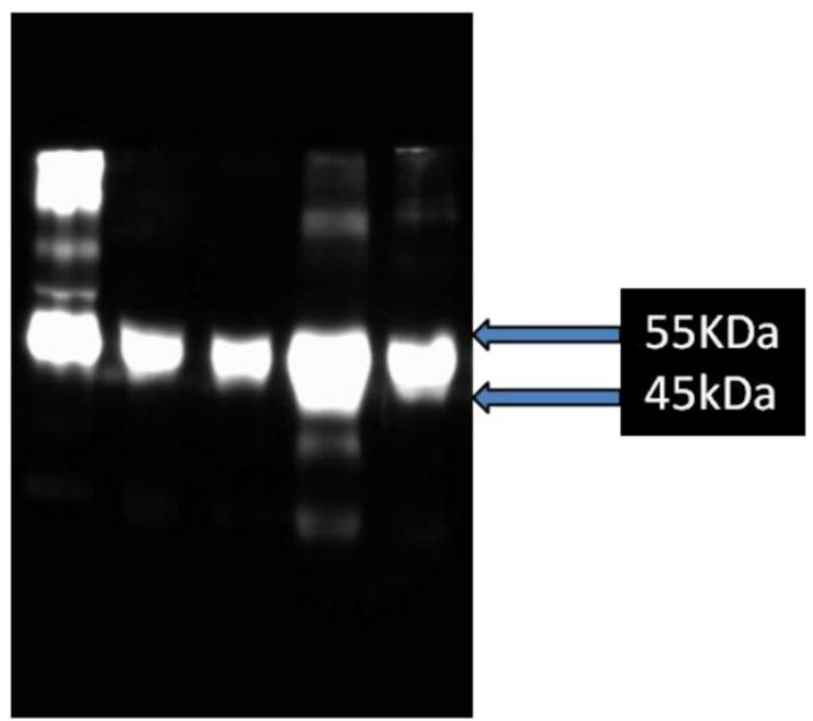

Figure 6. Western blot analysis after 10\% SDS-PAGE of AGP in bloods of patients pre-operatively compared to that from blood donors. Lane I, purified mucin from patient with pseudomyxoma peritonei, Lane 2, donor blood, Lanes 3-5, blood from patients undergoing gastrectomy for carcinoma of the stomach.

\section{Discussion}

This study was limited by the infrequency of surgical resections (inoperability because of advanced disease), low yields of purified mucin per sample, a lack of suitable normal tissue due to new forensic-legal regulations governing the use of tissue from cadavers and transplant donors and the inexplicable absence of the glycoprotein in some samples.

Mucins were extracted from crude mucus scrapings in denaturing media and the fractionation of the $40-50 \mathrm{kDa}$ glycoprotein with mucin during purification in a $\mathrm{CsCl}$ density gradient indicated that it was extensively glycosylated and could only be separated from mucins and albumin upon heating in SDS or by gel filtration. ${ }^{20}$ The glycoprotein was absent in crude mucus scrapings from cadaver and transplant donor stomachs. ${ }^{20}$

The close association of this glycoprotein with albumin, shown here and previously, ${ }^{17-20}$ even after two or sometimes three purification steps in a density gradient centrifugation in $\mathrm{CsCl}$ (Mall, personal communication), complicated attempts to isolate it in its pure form. Although stomachs of patients with gastric cancer are likely to have increased amounts of albu$\mathrm{min}$, through leakage of serum into the stomach, ${ }^{20,28}$ we are unable to explain why albumin was not removed during the purification process in $3.5 \mathrm{M} \mathrm{CsCl}$.

The $40-50 \mathrm{kDa}$ glycoprotein was best visualized by staining the gel with PAS, ${ }^{29}$ which then made it unsuitable for immunization (Bellstedt, personal communication). Ponceau S staining detected albumin and made easier the localization of the $40-50 \mathrm{kDa}$ glycoprotein, which always appeared immediately below albumin. To counteract reactivity with albumin (Figure 2A), the antibody was incubated with HSA for $1 \mathrm{~h}$ prior to electrophoresis. The reactivity of the antibody with larger mucins at the top of the running gel could be due to shared epitopes between them (Figure 2B, Lane 4), as shown previously 20,30 .

This study was more definitive than the previous ones ${ }^{17-20}$ in that those studies relied on the investigation of this glycoprotein in crude mucosal scrapings and gastric juice, ${ }^{21}$ and did not distinguish between normal and tumour portions of the mucosa. Immunohistochemical findings showed the expression of the $40-50 \mathrm{kDa}$ glycoprotein in normal tissue, with increasing amounts in intestinal metaplasia and cancer. Parietal cell staining in normal gastric mucosa was the first surprising hint that this glycoprotein may not be a mucin, as thought previously. ${ }^{20}$ In intestinal metaplasia and normal small intestine, the glycoprotein was found in the cytoplasm of columnar cells, and not in the mucus secreting goblet cells. The lack of tissue prevented us from examining the expression of the glycoprotein in all forms of metaplasia. In types I and II IM however, there was no difference in the expression of the glycoprotein. In this study and the previous one ${ }^{20}$ the cancers were mostly of the intestinal and diffuse type carcinomas with fewer signet, mucinous and mixed intestinal and diffuse type carcinomas.

Our results also confirmed previous findings of the cellular and tissue distribution of MUC5AC in the surface epithelium, ${ }^{6}$ MUC6 in the deep mucus glands and mucus neck cells ${ }^{5}$, MUC1 in the mucus cells of the surface epithelium and in the glands of the body ${ }^{2}$ and MUC4 in normal gastric mucosa. ${ }^{3}$ There was no expression of MUC1core and MUC2 in the normal gastric mucosa.

The combination of 2D gel electrophoresis and MALDI-TOF MS analyses led to a successful identification of this glycoprotein as AGP. An amino acid

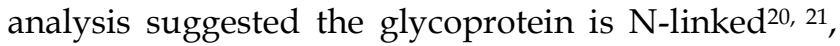
thus supporting its identification as AGP, which has been reported as a marker of disease in the blood of patients with carcinoma of the lung, breast, ovary 31,32 and liver. ${ }^{33}$ 
The immunomodulatory functions of AGP are physiological mechanisms meant to dampen the immune response and prevent unnecessary damage to nearby tissues during acute inflammation. ${ }^{34}$ However, during malignant transformation, the presence of AGP may confer unfair advantages to neoplastic cells such as protecting cells from apoptosis induced by inflammation. ${ }^{35}$ Aberrant glycosylation of AGP suggests it to be a strong candidate as a marker of the progression and prognosis of various cancers, with various glycoforms containing highly fucosylated triand tetra-antennary oligosaccharide side-chains at increased levels long after surgery, indicative of a poor prognosis. ${ }^{36}$ The role of AGP in the pathogenesis of gastric cancer is uncertain and its importance as a marker for pre-malignant disease requires further investigation. However, preliminary studies have shown that there is a slight increase in the blood levels of AGP in patients prior to and soon after surgery. This observation is being further explored with the aim of following up a larger group of patients for longer periods.

Although the CBB stained spots 1, 2 and 3 (Figure 4) were positively identified, tryptic digest peptides from spot 4 produced no positive hit in the protein databases, suggesting that its extensive glycosylation or PAS staining hindered digestion by trypsin (results not shown). Either of these factors could have caused chemical mass shifts which affected the measured protein/peptide mass. Measured tryptic peptide masses obtained for spots 1, 2 and 3 are very similar to those obtained from spot 4 , particularly for three peptides that matched to alpha-1-acid glycoprotein 1 precursor. This suggests that spot 4 might be a more glycosylated isoform of this protein.

The isoelectric point $(\mathrm{pI})$ of the identified alpha-1-acid glycoprotein (AGP), predicted in silico based on the amino acid sequence is 4.93 . This is congruent with the pI 3-4 observed in our study, and the slight shift is most likely due to post-translational modifications (Figure 5; Table1). On the other hand, the observed $50 \mathrm{kDa}$ molecular weight is twice the theoretical prediction $(23 \mathrm{kDa})$. Fifty percent of AGP is glycosylated ${ }^{37}$ and when treated with $\mathrm{N}$-glycosidase $\mathrm{F}$ its molecular weight is reduced to $23 \mathrm{kDa}$.

Identification of the glycoprotein as AGP, facilitates a study of its suitability as a clinical marker for pre-malignancy in a high-risk population in the Western Cape region of South Africa. We have begun a study to detect its levels in the blood of patients with cancer. Alternative MS compatible glycoprotein staining methods to visualize and identify spot 4 are also underway.

\section{Abbreviations:}

CsCl: Caesium chloride; IM: Intestinal metaplasia; PAS: Periodic acid Schiff; SDS-PAGE: Sodium dodecyl sulphate polyacrylamide gel electrophoresis; $\mathrm{HCl}$ : Hydrochloric acid; EDTA: ethylene diamine tetra acetic acid; NEM-N: ethylmaleimide; PMSF: phenylmethylsulfonylfluoride; DTT: dithiothreitol; GuHCl: guanidinium hydrochloride; IEF: Isoelectric focusing; AGP: Alpha-1: acid glycoprotein; CBB: Coomassie Brilliant Blue; MS: Mass spectrometry; MALDI-TOF: Matrix assisted laser desorption/ionization:time of flight; AB: Alcian Blue; HID: High iron diamine.

\section{Acknowledgements}

This study was supported by the University of Cape Town Research Fund, National Research Foundation (NRF) of South Africa and the South African Medical Research Council. We thank the D Floor Surgical Theatre Staff, at Groote Schuur Hospital and University of Cape Town Private Hospital, for helping with the handling of gastrectomy specimens and Ms Mary Wolfe for kindly providing the tissue blocks for immunohistochemical studies. The Western Province Blood Transfusion Service provided bloods from healthy donors.

\section{Conflict of Interest}

The authors have declared that no conflict of interest exists.

\section{References}

1. Kerss S, Allen A, Garner A. A simple method for measuring thickness of the mucus gel layer adherent to rat, frog and human gastric mucosa: Influence of feeding, prostaglandin, N-acetylcysteine and other agents. Clin. Sci. (Lond). 1982;63:187-195.

2. Reis CA, David L, Seixas M, Burchell J, Sobrinho-Simoes M. Expression of fully and under-glycosylated forms of MUC1 mucin in gastric carcinoma. Int. J. Cancer 1998;79:402-10.

3. Taylor KL, Mall AS, Barnard RA, Ho SB, Cruse JP. Immunohistochemical detection of gastric mucin in normal and disease states. Oncol. Res. 1998;10:465-73.

4. Ho SB, Shekels LL, Toribara NW, Kim YS, Lyftogt C, Cherwitz $\mathrm{DL}$, et al. Mucin gene expression in normal, preneoplastic, and neoplastic human gastric epithelium. Cancer Res. 1995;55:2681-90.

5. De Bolos C, Garrido M, Real FX. Muc6 apomucin shows a distinct normal tissue that correlates with lewis antigen expression in the human stomach. Gastroenterology. 1995;109:723-734.

6. Reis CA, David L, Nielsen PA, Clausen H, Mirgorodskaya K, Roepstorff $\mathrm{P}$, Sobrinho-Simoes M. Immunohistochemical study of MUC5AC expression in human gastric carcinomas using a novel monoclonal antibody. Int. J. Cancer 1997;74:112-21.

7. Allen A, Flemstrom G, Garner A, Kivilaakso E. Gastroduodenal mucosal protection. Physiol. Rev. 1993;73:823-57.

8. Parkin DM, Bray FI, Devesa SS. Cancer burden in the year 2000. The global picture. Eur J Cancer 2001;37(Suppl 8):S4-66. 
9. Botha MC. Blood group gene frequencies. An indication of the genetic constitution of population samples in Cape Town. Am J Roentgenol Radium Thr Nucl Med. 1972;115(Suppl):1-27.

10. Bradshaw E, Harington JS. The changing pattern of cancer mortality in South Africa, 1949-1969. S. Afr. Med. J. 1975;49:919-25.

11. Wyndham $\mathrm{CH}$. Comparison and ranking of cancer mortality rates in the various populations of the RSA in 1970. S. Afr. Med. J. 1985;67:584-7.

12. Magnani JL, Steplewski Z, Koprowski H, Ginsburg V. Identification of the gastrointestinal and pancreatic cancer-associated antigen detected by monoclonal antibody 19-9 in the sera of patients as a mucin. Cancer Res. 1983;43:5489-92.

13. Fuchs CS, Mayer RJ. Gastric carcinoma. N. Engl. J. Med. 1995:333:32-41.

14. Inagaki $\mathrm{H}$, Sakamoto J, Nakazato $\mathrm{H}$, Bishop AE, Yura J. Expression of Lewis(a), Lewis(b), and sialated Lewis(a) antigens in early and advanced human gastric cancers. J. Surg. Oncol. 1990;44:208-13.

15. Devine PL, McKenzie FC. Mucins, structure, function and association with malignancy. Bioessays 1992;14:619-25.

16. Graham RA, Burchell JM, Taylor-Papadimitriou J. The polymorphic epithelial mucin: potential as an immunogen for a cancer vaccine. Cancer Immunol. Immunother. 1996;42:71-80.

17. Mall AS, McLeod H, Jaskiewicz K, Dent DM, Hickman R. Putative glycosylated 'linker' protein in mucin in gastric carcinoma. S. Afr. J. Sci. 1990;86:45-46.

18. Mall AS, Hickman R, Dent D. Further investigation of mucins in gastric carcinoma. South African Journal of Science 1992;88:233-234.

19. Mall A, McLeod H, Dent D, Hickman R. Gastric cancer mucins as clinical markers. Gut 1992;33:1681.

20. Mall AS, McLeod HA, Hickman R, Kahn D, Dent DM. Fragmentation pattern of mucins in normal and diseased gastric mucosae: a glycoprotein fractionates with gastric mucins purified from mucosal scrapings of cancer and peptic ulcer patients. Digestion 1999;60:216-26.

21. Mall AS, McConney Z, Lotz Z, Tyler M, Mcleod H, Hickman R, et al. Increased fragmentation of MUC5AC mucins in gastric juice of patients with ulceration and carcinoma. S. Afr. J. Sci. 2000;96:39-43.

22. Hakkinen I, Nevalainen T, Paasivuo R, Partanen P, Seppala K, Sipponen P. Gastric cancer associated structure in mucus glycoproteins shown as a clinically useful marker. Gut 1991:32:1465-9.

23. Creeth JM, Denborough MA. Density gradient equilibrium methods applied to blood-group specific glycoproteins. FEBS Lett. 1970;6:117-120.

24. Mall AS, Chirwa N, Govender D, Lotz Z, Tyler M, Rodrigue G, Kahn D and Goldberg P. MUC2, MUC5AC and MUC5B in the mucus of a patient with pseudomyxoma peritonei: Biochemical and immunohistochemical study. Pathology International 2007; 57(8):537-547.

25. Bellstedt DU, Human PA, Rowland GF, Van der Merwe KJ. Acid-treated, naked bacteria as immune carriers for protein antigens. J. Immunol. Methods 1987;98:249-55.

26. Mall AS, Lotz Z, Tyler M, Goldberg P, Rodrigues J, Kahn D, et al. Immunohistochemical and Biochemical Characterization of Mucin in Pseudomyxoma Peritonei: A Case Study. Gastroenterol. 2011;5:5-16.

27. Thomas LA, Majimi JS, du Preez MG, Rees JG, Ndimba BK. Establishment of proteome spot profiles and comparative analysis of red and green phenotypes of 'Bon Rouge' pear (Pyrus communis L.) leaves. African $J$ of Biotechnol. 2010;9(28):4334-4341.

28. Yamaoka K, Noguchi S, Higashi H, Kinnaid D. Studies on the Mechanism of Hypoproteinemia in Gastric Cancer: Albumin
Leakage in to Gastric Juice in Rats with N-MethylN'-Nitro-N-Nitrosoguanidine Induced Gastric Cancer. Japaneses Journal of Clinical Oncology 1974;4:105-113.

29. Dubray G, Bezard G. A highly sensitive periodic acid-silver stain for 1,2-diol groups of glycoproteins and polysaccharides in polyacrylamide gels. Anal. Biochem. 1982;119:325-9.

30. Bara J, Gautier R, LePendu J, Oriol R. Immunochemical characterization of mucins. Biochem. J. 1998;254:185-193.

31. Tosner J, Krejsek J, Louda B. Serum prealbumin, transferrin and alpha-1-acid glycoprotein in patients with gynecological carcinomas. Neoplasma. 1988;35:403-411.

32. Blain PG, Mucklow JC, Rawlins MD, Roberts DF, Routledge PA, Shand DG. Determinants of plasma alpha 1-acid glycoprotein (aag) concentrations in health. Br. J. Clin. Pharmacol. 1985;20:500-502.

33. Song EY, Kim KA, Kim YD, Lee EY, Lee HS, Kim HJ et al. Elevation of serum asialo-alpha(1) acid glycoprotein concentration in patients with hepatic cirrhosis and hepatocellular carcinoma as measured by antibody-lectin sandwich assay. Hepatol. Res. 2003;26:311-317.

34. Hochepied T, Berger FG, Baumann H, Libert C. Alpha(1)-acid glycoprotein: An acute phase protein with inflammatory and immunomodulating properties. Cytokine Growth Factor Rev. 2003;14:25-34.

35. Ceciliani F, Pocacqua V, Miranda-Ribera A, Bronzo V, Lecchi C, Sartorelli P. Alpha(1)-acid glycoprotein modulates apoptosis in bovine monocytes. Vet. Immunol. Immunopathol. 2007;116:145-152.

36. Hashimoto S, Asao T, Takahashi J, Yagihashi Y, Nishimura T, Saniabadi AR et al. Alpha1-acid glycoprotein fucosylation as a marker of carcinoma progression and prognosis. Cancer. 2004;101:2825-2836.

37. Nakamura O, Nozawa Y, Saito E, Ikeda D, Tsutsui S. An alpha-1 acid glycoprotein-like protein as a major component of the ovarian cavity fluid of viviparous fish, neoditrema ransonnetti (perciformes, embiotocidae). Comp. Biochem. Physiol. A Mol. Integr. Physiol. 2009;153:222-229. 